\title{
Informed consent procedures in patients with an acute inability to provide informed consent: Policy and practice in the CENTER-TBI study
}

Roel P.J. van Wijk ${ }^{\mathrm{a}, 1}$, Jeroen T.J.M. van Dijck ${ }^{\mathrm{a}, 1}$, Marjolein Timmers ${ }^{\mathrm{b}}$, Ernest van Veen ${ }^{\mathrm{b}, \mathrm{c}}$, Giuseppe Citerio ${ }^{\mathrm{d}, \mathrm{e}}$, Hester F. Lingsma c , Andrew I.R. Maas ${ }^{\text {f,g }}$, David K. Menon ${ }^{\text {h }}$, Wilco C. Peul ${ }^{\mathrm{a}}$, Nino Stocchetti ${ }^{\mathrm{i}, \mathrm{j}}$, Erwin J.O. Kompanje ${ }^{\mathrm{b}, \mathrm{k}, *}$,The CENTER-TBI investigators and participants:

Cecilia Åkerlund ${ }^{1}$, Krisztina Amrein ${ }^{2}$, Nada Andelic ${ }^{3}$, Lasse Andreassen ${ }^{4}$, Audny Anke ${ }^{5}$, Anna Antoni ${ }^{6}$, Gérard Audibert ${ }^{7}$, Philippe Azouvi ${ }^{8}$, Maria Luisa Azzolini ${ }^{9}$, Ronald Bartels ${ }^{10}$, Pál Barzó ${ }^{11}$, Romuald Beauvais ${ }^{12}$, Ronny Beer ${ }^{13}$, Bo-Michael Bellander ${ }^{14}$, Antonio Belli ${ }^{15}$, Habib Benali ${ }^{16}$, Maurizio Berardino ${ }^{17}$, Luigi Beretta ${ }^{9}$, Morten Blaabjerg ${ }^{18}$, Peter Bragge ${ }^{19}$, Alexandra Brazinova ${ }^{20}$, Vibeke Brinck ${ }^{21}$, Joanne Brooker ${ }^{22}$, Camilla Brorsson ${ }^{23}$, Andras Buki ${ }^{24}$, Monika Bullinger ${ }^{25}$, Manuel Cabeleira ${ }^{26}$, Alessio Caccioppola ${ }^{27}$, Emiliana Calappi ${ }^{27}$, Maria Rosa Calvi ${ }^{9}$, Peter Cameron ${ }^{28}$, Guillermo Carbayo Lozano ${ }^{29}$, Marco Carbonara ${ }^{27}$, Simona Cavallo ${ }^{17}$, Giorgio Chevallard ${ }^{30}$, Arturo Chieregato ${ }^{30}$, Giuseppe Citerio ${ }^{31,32}$, Iris Ceyisakar ${ }^{33}$, Mark Coburn $^{34}$, Jonathan Coles ${ }^{35}$, Jamie D. Cooper ${ }^{36}$, Marta Correia ${ }^{37}$, Amra Čović ${ }^{38}$, Nicola Curry ${ }^{39}$, Endre Czeiter ${ }^{24}$, Marek Czosnyka ${ }^{26}$, Claire Dahyot-Fizelier ${ }^{40}$, Paul Dark ${ }^{41}$, Helen Dawes ${ }^{42}$, Véronique De Keyser ${ }^{43}$, Vincent Degos ${ }^{16}$, Francesco Della Corte ${ }^{44}$, Hugo den Boogert ${ }^{10}$, Bart Depreitere ${ }^{45}$, Đula Đilvesi ${ }^{46}$, Abhishek Dixit ${ }^{47}$, Emma Donoghue ${ }^{22}$, Jens Dreier ${ }^{48}$, Guy-Loup Dulière ${ }^{49}$, Ari Ercole ${ }^{47}$, Patrick Esser ${ }^{42}$, Erzsébet Ezer ${ }^{50}$, Martin Fabricius ${ }^{51}$, Valery L. Feigin ${ }^{52}$, Kelly Foks ${ }^{53}$, Shirin Frisvold ${ }^{54}$, Alex Furmanov ${ }^{55}$, Pablo Gagliardo ${ }^{56}$, Damien Galanaud ${ }^{16}$, Dashiell Gantner ${ }^{28}$, Guoyi Gao ${ }^{57}$, Pradeep George ${ }^{58}$ , Alexandre Ghuysen ${ }^{59}$, Lelde Giga ${ }^{60}$, Ben Glocker ${ }^{61}$, Jagoš Golubovic ${ }^{46}$, Pedro A. Gomez ${ }^{62}$, Johannes Gratz ${ }^{63}$, Benjamin Gravesteijn ${ }^{33}$, Francesca Grossi ${ }^{44}$, Russell L. Gruen ${ }^{64}$, Deepak Gupta ${ }^{65}$, Juanita A. Haagsma ${ }^{33}$, Iain Haitsma ${ }^{66}$, Raimund Helbok ${ }^{13}$, Eirik Helseth ${ }^{67}$, Lindsay Horton ${ }^{68}$, Jilske Huijben ${ }^{33}$, Peter J. Hutchinson ${ }^{69}$, Bram Jacobs $^{70}$, Stefan Jankowski ${ }^{71}$, Mike Jarrett ${ }^{21}$, Ji-Yao Jiang ${ }^{57}$, Faye Johnson ${ }^{72}$, Kelly Jones ${ }^{52}$, Mladen Karan ${ }^{46}$, Angelos G. Kolias ${ }^{69}$, Erwin Kompanje ${ }^{73}$, Daniel Kondziella ${ }^{51}$, Evgenios Koraropoulos ${ }^{47}$, Lars-Owe Koskinen ${ }^{74}$, Noémi Kovács ${ }^{75}$, Ana Kowark ${ }^{34}$, Alfonso Lagares ${ }^{62}$, Linda Lanyon ${ }^{58}$, Steven Laureys ${ }^{76}$, Fiona Lecky ${ }^{77,78}$, Didier Ledoux ${ }^{76}$, Rolf Lefering ${ }^{79}$, Valerie Legrand ${ }^{80}$, Aurelie Lejeune ${ }^{81}$, Leon Levi ${ }^{82}$, Roger Lightfoot ${ }^{83}$, Hester Lingsma ${ }^{33}$, Andrew I.R. Maas ${ }^{43}$, Ana M. Castaño-León ${ }^{62}$, Marc Maegele ${ }^{84}$, Marek Majdan ${ }^{20}$, Alex Manara ${ }^{85}$, Geoffrey Manley ${ }^{86}$, Costanza Martino ${ }^{87}$, Hugues Maréchal ${ }^{49}$, Julia Mattern ${ }^{88}$ , Catherine McMahon ${ }^{89}$, Béla Melegh ${ }^{90}$, David Menon ${ }^{47}$, Tomas Menovsky ${ }^{43}$, Benoit Misset ${ }^{76}$, Davide Mulazzi ${ }^{27}$, Visakh Muraleedharan ${ }^{58}$, Lynnette Murray ${ }^{28}$, Ancuta Negru ${ }^{91}$, David Nelson ${ }^{1}$, Virginia Newcombe ${ }^{47}$, Daan Nieboer ${ }^{33}$, József Nyirádi ${ }^{2}$, Otesile Olubukola ${ }^{77}$, Matej Oresic ${ }^{92}$, Fabrizio Ortolano ${ }^{27}$, Aarno Palotie ${ }^{93,94,95}$, Paul M. Parizel ${ }^{96}$, Jean-François Payen ${ }^{97}$, Natascha Perera ${ }^{12}$, Vincent Perlbarg ${ }^{16}$, Paolo Persona ${ }^{98}$, Wilco Peul ${ }^{99,144}$, Anna Piippo-Karjalainen ${ }^{100}$, Matti Pirinen ${ }^{93}$, Horia Ples ${ }^{91}$, Suzanne Polinder ${ }^{33}$, Inigo Pomposo ${ }^{29}$, Jussi P. Posti ${ }^{101}$, Louis Puybasset ${ }^{102}$, Andreea Radoi ${ }^{103}$, Arminas Ragauskas ${ }^{104}$, Rahul Raj ${ }^{100}$, Malinka Rambadagalla ${ }^{105}$, Jonathan Rhodes ${ }^{106}$, Sylvia Richardson ${ }^{107}$, Sophie Richter ${ }^{47}$, Samuli Ripatti ${ }^{93}$, Saulius Rocka ${ }^{104}$, Cecilie Roe ${ }^{108}$, Olav Roise ${ }^{109,110}$, Jonathan Rosand ${ }^{111}$,

Abbreviations: TBI, Traumatic Brain Injury; IRB, Institutional Review Board; EU, European Union; ER, Emergency Room; ICU, Intensive Care Unit; INCF, International Neuroinformatics Coordinating Facility; UK, United Kingdom.

* Corresponding author at: Department of Intensive Care Medicine, Erasmus MC University Medical Center, P.0. Box 2040, 3000 CA Rotterdam, the Netherlands.

E-mail address: e.j.o.kompanje@erasmusmc.nl (E.J.O. Kompanje).

${ }^{1}$ Contributed equally to the study. 
Jeffrey V. Rosenfeld ${ }^{112}$, Christina Rosenlund ${ }^{113}$, Guy Rosenthal ${ }^{55}$, Rolf Rossaint ${ }^{34}$, Sandra Rossi ${ }^{98}$, Daniel Rueckert ${ }^{61}$, Martin Rusnák ${ }^{114}$, Juan Sahuquillo ${ }^{103}$, Oliver Sakowitz ${ }^{88,115}$, Renan Sanchez-Porras ${ }^{115}$, Janos Sandor ${ }^{116}$, Nadine Schäfer ${ }^{79}$, Silke Schmidt ${ }^{117}$, Herbert Schoechl ${ }^{118}$, Guus Schoonman ${ }^{119}$, Rico Frederik Schou ${ }^{120}$, Elisabeth Schwendenwein ${ }^{6}$, Charlie Sewalt ${ }^{33}$, Toril Skandsen ${ }^{121,122}$, Peter Smielewski ${ }^{26}$, Abayomi Sorinola ${ }^{123}$, Emmanuel Stamatakis ${ }^{47}$, Simon Stanworth ${ }^{39}$, Robert Stevens ${ }^{124}$, William Stewart ${ }^{125}$, Ewout W. Steyerberg ${ }^{33,126}$, Nino Stocchetti ${ }^{127}$, Nina Sundström ${ }^{128}$, Anneliese Synnot ${ }^{22,129}$, Riikka Takala ${ }^{130}$, Viktória Tamás ${ }^{123}$, Tomas Tamosuitis ${ }^{131}$, Mark Steven Taylor ${ }^{20}$, Braden Te Ao ${ }^{52}$, Olli Tenovuo ${ }^{101}$, Alice Theadom ${ }^{52}$, Matt Thomas ${ }^{85}$, Dick Tibboel ${ }^{132}$, Marjolein Timmers ${ }^{73}$, Christos Tolias ${ }^{133}$, Tony Trapani ${ }^{28}$, Cristina Maria Tudora ${ }^{91}$, Peter Vajkoczy ${ }^{134}$, Shirley Vallance ${ }^{28}$, Egils Valeinis ${ }^{60}$, Zoltán Vámos ${ }^{50}$, Gregory Van der Steen ${ }^{43}$, Joukje van der Naalt ${ }^{70}$, Jeroen T.J.M. van Dijck ${ }^{99,144}$, Thomas A. van Essen ${ }^{99,144}$, Wim Van Hecke ${ }^{135}$, Caroline van Heugten ${ }^{42}$, Dominique Van Praag ${ }^{137}$, Thijs Vande Vyvere ${ }^{135}$, Roel P.J. van Wijk ${ }^{99,144}$, Alessia Vargiolu ${ }^{32}$, Emmanuel Vega ${ }^{81}$, Kimberley Velt ${ }^{33}$, Jan Verheyden ${ }^{135}$, Paul M. Vespa ${ }^{138}$, Anne Vik ${ }^{120,139}$, Rimantas Vilcinis ${ }^{131}$, Victor Volovici ${ }^{66}$, Nicole von Steinbüchel ${ }^{38}$, Daphne Voormolen ${ }^{33}$, Petar Vulekovic ${ }^{46}$, Kevin K.W. Wang ${ }^{140}$, Eveline Wiegers ${ }^{33}$, Guy Williams ${ }^{47}$, Lindsay Wilson ${ }^{68}$, Stefan Winzeck ${ }^{47}$, Stefan Wolf ${ }^{141}$, Zhihui Yang ${ }^{140}$, Peter Ylén ${ }^{142}$, Alexander Younsi ${ }^{88}$, Frederick A. Zeiler ${ }^{47,143}$, Veronika Zelinkova ${ }^{20}$, Agate Ziverte $^{60}$, Tommaso Zoerle ${ }^{27}$

${ }^{1}$ Department of Physiology and Pharmacology, Section of Perioperative Medicine and Intensive Care, Karolinska Institutet, Stockholm, Sweden

2 János Szentágothai Research Centre, University of Pécs, Pécs, Hungary

${ }^{3}$ Division of Surgery and Clinical Neuroscience, Department of Physical Medicine and Rehabilitation, Oslo University Hospital and University of Oslo, Oslo, Norway

${ }^{4}$ Department of Neurosurgery, University Hospital Northern Norway, Tromso, Norway

${ }^{5}$ Department of Physical Medicine and Rehabilitation, University Hospital Northern Norway, Tromso, Norway

${ }^{6}$ Trauma Surgery, Medical University Vienna, Vienna, Austria

${ }^{7}$ Department of Anesthesiology E' Intensive Care, University Hospital Nancy, Nancy, France

${ }^{8}$ Raymond Poincare hospital, Hopitaux de Paris, Paris, France

${ }^{9}$ Department of Anesthesiology E' Intensive Care, S Raffaele University Hospital, Milan, Italy

${ }^{10}$ Department of Neurosurgery, Radboud University Medical Center, Nijmegen, the Netherlands

${ }^{11}$ Department of Neurosurgery, University of Szeged, Szeged, Hungary

12 International Projects Management, ARTTIC, Munchen, Germany

${ }^{13}$ Department of Neurology, Neurological Intensive Care Unit, Medical University of Innsbruck, Innsbruck, Austria

${ }^{14}$ Department of Neurosurgery \& Anesthesia \&' intensive care medicine, Karolinska University Hospital, Stockholm, Sweden

${ }^{15}$ NIHR Surgical Reconstruction and Microbiology Research Centre, Birmingham, UK

${ }^{16}$ Anesthesie-Réanimation, Hopitaux de Paris, Paris, France

17 Department of Anesthesia E' ICU, AOU Città della Salute e della Scienza di Torino - Orthopedic and Trauma Center, Torino, Italy

${ }^{18}$ Department of Neurology, Odense University Hospital, Odense, Denmark

${ }^{19}$ BehaviourWorks Australia, Monash Sustainability Institute, Monash University, Victoria, Australia

${ }^{20}$ Department of Public Health, Faculty of Health Sciences and Social Work, Trnava University, Trnava, Slovakia

21 Quesgen Systems Inc, Burlingame, CA, USA

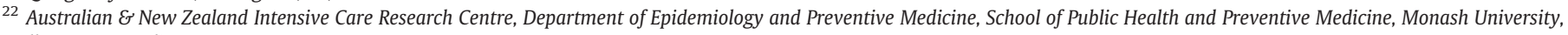
Melbourne, Australia

${ }^{23}$ Department of Surgery and Perioperative Science, Umeå University, Umeå, Sweden

24 Department of Neurosurgery, Medical School, Hungary and Neurotrauma Research Group, János Szentágothai Research Centre, University of Pécs, Hungary

25 Department of Medical Psychology, Universitätsklinikum Hamburg-Eppendorf, Hamburg, Germany

${ }^{26}$ Brain Physics Lab, Division of Neurosurgery, Dept of Clinical Neurosciences, University of Cambridge, Addenbrooke's Hospital, Cambridge, UK

${ }^{27}$ Neuro ICU, Fondazione IRCCS Cà Granda Ospedale Maggiore Policlinico, Milan, Italy

${ }^{28}$ ANZIC Research Centre, Monash University, Department of Epidemiology and Preventive Medicine, Melbourne, Victoria, Australia

${ }^{29}$ Department of Neurosurgery, Hospital of Cruces, Bilbao, Spain

${ }^{30}$ NeuroIntensive Care, Niguarda Hospital, Milan, Italy

${ }^{31}$ School of Medicine and Surgery, Università Milano Bicocca, Milano, Italy

32 NeuroIntensive Care, ASST di Monza, Monza, Italy

${ }^{33}$ Department of Public Health, Erasmus Medical Center, University Medical Center, Rotterdam, the Netherlands

${ }^{34}$ Department of Anaesthesiology, University Hospital of Aachen, Aachen, Germany

${ }^{35}$ Department of Anesthesia \&' Neurointensive Care, Cambridge University Hospital NHS Foundation Trust, Cambridge, UK

${ }^{36}$ School of Public Health \& PM, Monash University and The Alfred Hospital, Melbourne, Victoria, Australia

${ }^{37}$ Radiology/MRI department, MRC Cognition and Brain Sciences Unit, Cambridge, UK

${ }^{38}$ Institute of Medical Psychology and Medical Sociology, Universitätsmedizin Göttingen, Göttingen, Germany

39 Oxford University Hospitals NHS Trust, Oxford, UK

${ }^{40}$ Intensive Care Unit, CHU Poitiers, Potiers, France

${ }^{41}$ University of Manchester NIHR Biomedical Research Centre, Critical Care Directorate, Salford Royal Hospital NHS Foundation Trust, Salford, UK

${ }^{42}$ Movement Science Group, Faculty of Health and Life Sciences, Oxford Brookes University, Oxford, UK

${ }^{43}$ Department of Neurosurgery, Antwerp University Hospital and University of Antwerp, Edegem, Belgium

${ }^{44}$ Department of Anesthesia E' Intensive Care, Maggiore Della Carità Hospital, Novara, Italy

${ }^{45}$ Department of Neurosurgery, University Hospitals Leuven, Leuven, Belgium

${ }^{46}$ Department of Neurosurgery, Clinical centre of Vojvodina, Faculty of Medicine, University of Novi Sad, Novi Sad, Serbia

${ }^{47}$ Division of Anaesthesia, University of Cambridge, Addenbrooke's Hospital, Cambridge, UK

${ }^{48}$ Center for Stroke Research Berlin, Charité, Universitätsmedizin Berlin, Freie Universität Berlin, Humboldt-Universität zu Berlin, Berlin Institute of Health, Berlin, Germany

49 Intensive Care Unit, CHR Citadelle, Liège, Belgium

${ }^{50}$ Department of Anaesthesiology and Intensive Therapy, University of Pécs, Pécs, Hungary

${ }^{51}$ Departments of Neurology, Clinical Neurophysiology and Neuroanesthesiology, Region Hovedstaden Rigshospitalet, Copenhagen, Denmark

${ }^{52}$ National Institute for Stroke and Applied Neurosciences, Faculty of Health and Environmental Studies, Auckland University of Technology, Auckland, New Zealand

53 Department of Neurology, Erasmus MC, Rotterdam, the Netherlands

${ }^{54}$ Department of Anesthesiology and Intensive care, University Hospital Northern Norway, Tromso, Norway 
55 Department of Neurosurgery, Hadassah-hebrew University Medical center, Jerusalem, Israel

${ }^{56}$ Fundación Instituto Valenciano de Neurorrehabilitación (FIVAN), Valencia, Spain

${ }^{57}$ Department of Neurosurgery, Shanghai Renji hospital, Shanghai Jiaotong University/school of medicine, Shanghai, China

${ }^{58}$ Karolinska Institutet, INCF International Neuroinformatics Coordinating Facility, Stockholm, Sweden

${ }^{59}$ Emergency Department, $\mathrm{CHU}$, Liège, Belgium

${ }^{60}$ Neurosurgery clinic, Pauls Stradins Clinical University Hospital, Riga, Latvia

${ }^{61}$ Department of Computing, Imperial College London, London, UK

62 Department of Neurosurgery, Hospital Universitario 12 de Octubre, Madrid, Spain

${ }^{63}$ Department of Anesthesia, Critical Care and Pain Medicine, Medical University of Vienna, Austria

${ }^{64}$ College of Health and Medicine, Australian National University, Canberra, Australia

${ }^{65}$ Department of Neurosurgery, Neurosciences Centre E JPN Apex trauma centre, All India Institute of Medical Sciences, New Delhi 110029, India

${ }^{66}$ Department of Neurosurgery, Erasmus MC, Rotterdam, the Netherlands

${ }^{67}$ Department of Neurosurgery, Oslo University Hospital, Oslo, Norway

${ }^{68}$ Division of Psychology, University of Stirling, Stirling, UK

${ }^{69}$ Division of Neurosurgery, Department of Clinical Neurosciences, Addenbrooke's Hospital \& University of Cambridge, Cambridge, UK

${ }^{70}$ Department of Neurology, University of Groningen, University Medical Center Groningen, Groningen, the Netherlands

${ }^{71}$ Neurointensive Care, Sheffield Teaching Hospitals NHS Foundation Trust, Sheffield, UK

72 Salford Royal Hospital NHS Foundation Trust Acute Research Delivery Team, Salford, UK

${ }^{73}$ Department of Intensive Care, Department of Ethics and Philosophy of Medicine, Erasmus Medical Center, Rotterdam, the Netherlands

74 Department of Clinical Neuroscience, Neurosurgery, Umeå University, Umeå, Sweden

${ }^{75}$ Hungarian Brain Research Program - Grant No. KTIA_13_NAP-A-II/8, University of Pécs, Pécs, Hungary

${ }^{76}$ Cyclotron Research Center, University of Liège, Liège, Belgium

77 Centre for Urgent and Emergency Care Research (CURE), Health Services Research Section, School of Health and Related Research (ScHARR), University of Sheffield, Sheffield, UK

${ }^{78}$ Emergency Department, Salford Royal Hospital, Salford, UK

${ }^{79}$ Institute of Research in Operative Medicine (IFOM), Witten/Herdecke University, Cologne, Germany

${ }^{80}$ VP Global Project Management CNS, ICON, Paris, France

${ }^{81}$ Department of Anesthesiology-Intensive Care, Lille University Hospital, Lille, France

82 Department of Neurosurgery, Rambam Medical Center, Haifa, Israel

83 Department of Anesthesiology \& Intensive Care, University Hospitals Southhampton NHS Trust, Southhampton, UK

${ }^{84}$ Cologne-Merheim Medical Center (CMMC), Department of Traumatology, Orthopedic Surgery and Sportmedicine, Witten/Herdecke University, Cologne, Germany

${ }^{85}$ Intensive Care Unit, Southmead Hospital, Bristol, Bristol, UK

${ }^{86}$ Department of Neurological Surgery, University of California, San Francisco, CA, USA

${ }^{87}$ Department of Anesthesia \&' Intensive Care, M. Bufalini Hospital, Cesena, Italy

${ }^{88}$ Department of Neurosurgery, University Hospital Heidelberg, Heidelberg, Germany

${ }^{89}$ Department of Neurosurgery, The Walton centre NHS Foundation Trust, Liverpool, UK

90 Department of Medical Genetics, University of Pécs, Pécs, Hungary

${ }^{91}$ Department of Neurosurgery, Emergency County Hospital Timisoara, Timisoara, Romania

92 School of Medical Sciences, Örebro University, Örebro, Sweden

${ }_{93}$ Institute for Molecular Medicine Finland, University of Helsinki, Helsinki, Finland

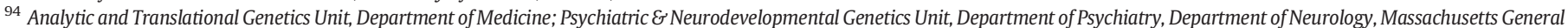
Hospital, Boston, MA, USA

${ }_{95}$ Program in Medical and Population Genetics, The Stanley Center for Psychiatric Research, The Broad Institute of MIT and Harvard, Cambridge, MA, USA

${ }^{96}$ Department of Radiology, University of Antwerp, Edegem, Belgium

${ }^{97}$ Department of Anesthesiology \&' Intensive Care, University Hospital of Grenoble, Grenoble, France

${ }^{98}$ Department of Anesthesia E' Intensive Care, Azienda Ospedaliera Università di Padova, Padova, Italy

${ }^{99}$ Dept. of Neurosurgery, Leiden University Medical Center, Leiden, the Netherlands

${ }^{100}$ Department of Neurosurgery, Helsinki University Central Hospital, Finland

101 Division of Clinical Neurosciences, Department of Neurosurgery and Turku Brain Injury Centre, Turku University Hospital and University of Turku, Turku, Finland

102 Department of Anesthesiology and Critical Care, Pitié -Salpêtrière Teaching Hospital, Hôpitaux de Paris and University Pierre et Marie Curie, Paris, France

${ }^{103}$ Neurotraumatology and Neurosurgery Research Unit (UNINN), Vall d'Hebron Research Institute, Barcelona, Spain

104 Department of Neurosurgery, Kaunas University of technology and Vilnius University, Vilnius, Lithuania

105 Department of Neurosurgery, Rezekne Hospital, Latvia

106 Department of Anaesthesia, Critical Care E' Pain Medicine NHS Lothian, University of Edinburg, Edinburgh, UK

${ }^{107}$ MRC Biostatistics Unit, Cambridge Institute of Public Health, Cambridge, UK

108 Department of Physical Medicine and Rehabilitation, Oslo University Hospital/University of Oslo, Oslo, Norway

109 Division of Orthopedics, Oslo University Hospital, Oslo, Norway

${ }^{110}$ Institue of Clinical Medicine, Faculty of Medicine, University of Oslo, Oslo, Norway

111 Broad Institute, Cambridge MA, Harvard Medical School, Boston MA, Massachusetts General Hospital, Boston, MA, USA

112 National Trauma Research Institute, The Alfred Hospital, Monash University, Melbourne, Victoria, Australia

113 Department of Neurosurgery, Odense University Hospital, Odense, Denmark

114 International Neurotrauma Research Organisation, Vienna, Austria

115 Klinik für Neurochirurgie, Klinikum Ludwigsburg, Ludwigsburg, Germany

${ }^{116}$ Division of Biostatistics and Epidemiology, Department of Preventive Medicine, University of Debrecen, Debrecen, Hungary

117 Department Health and Prevention, University Greifswald, Greifswald, Germany

118 Department of Anaesthesiology and Intensive Care, AUVA Trauma Hospital, Salzburg, Austria

119 Department of Neurology, Elisabeth-TweeSteden Ziekenhuis, Tilburg, the Netherlands

120 Department of Neuroanesthesia and Neurointensive Care, Odense University Hospital, Odense, Denmark

${ }^{121}$ Department of Neuromedicine and Movement Science, Norwegian University of Science and Technology, NTNU, Trondheim, Norway

122 Department of Physical Medicine and Rehabilitation, St.Olavs Hospital, Trondheim University Hospital, Trondheim, Norway

123 Department of Neurosurgery, University of Pécs, Pécs, Hungary

124 Division of Neuroscience Critical Care, John Hopkins University School of Medicine, Baltimore, USA

125 Department of Neuropathology, Queen Elizabeth University Hospital, University of Glasgow, Glasgow, UK

126 Dept. of Department of Biomedical Data Sciences, Leiden University Medical Center, Leiden, the Netherlands

127 Department of Pathophysiology and Transplantation, Milan University, Neuroscience ICU, Fondazione IRCCS Cà Granda Ospedale Maggiore Policlinico, Milano, Italy

128 Department of Radiation Sciences, Biomedical Engineering, Umeå University, Umeå, Sweden

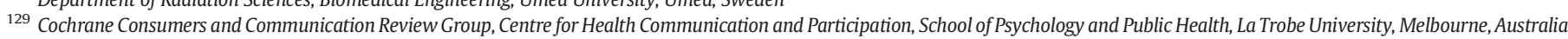

${ }^{130}$ Perioperative Services, Intensive Care Medicine and Pain Management, Turku University Hospital and University of Turku, Turku, Finland

131 Department of Neurosurgery, Kaunas University of Health Sciences, Kaunas, Lithuania

132 Intensive Care and Department of Pediatric Surgery, Erasmus Medical Center, Sophia Children's Hospital, Rotterdam, the Netherlands

133 Department of Neurosurgery, Kings college London, London, UK 
${ }^{134}$ Neurologie, Neurochirurgie und Psychiatrie, Charité - Universitätsmedizin Berlin, Berlin, Germany

135 icoMetrix NV, Leuven, Belgium

137 Psychology Department, Antwerp University Hospital, Edegem, Belgium

138 Director of Neurocritical Care, University of California, Los Angeles, USA

139 Department of Neurosurgery, St.Olavs Hospital, Trondheim University Hospital, Trondheim, Norway

140 Department of Emergency Medicine, University of Florida, Gainesville, FL, USA

${ }^{141}$ Department of Neurosurgery, Universitätsmedizin Berlin, Freie Universität Berlin, Humboldt-Universität zu Berlin, Berlin Institute of Health, Berlin, Germany

142 VTT Technical Research Centre, Tampere, Finland

143 Section of Neurosurgery, Department of Surgery, Rady Faculty of Health Sciences, University of Manitoba, Winnipeg, MB, Canada

144 Dept. of Neurosurgery, Medical Center Haaglanden, The Hague, the Netherlands

a University Neurosurgical Center Holland, LUMC, HMC \& HAGA, Leiden \& The Hague, the Netherlands

${ }^{\mathrm{b}}$ Department of Intensive Care, Erasmus MC - University Medical Centre Rotterdam, Rotterdam, the Netherlands

c Centre for Medical Decision Making, Department of Public Health, Erasmus MC - University Medical Centre Rotterdam, Rotterdam, the Netherlands

d School of Medicine and Surgery, University of Milan-Bicocca, Milan, Italy

e San Gerardo Hospital, ASST, Monza, Italy

${ }^{\mathrm{f}}$ Department of Neurosurgery, Antwerp University Hospital, Edegem, Belgium

gniversity of Antwerp, Antwerp, Belgium

h Department of Anaesthesia, University of Cambridge, Cambridge, United Kingdom

i Department of Physiopathology and Transplantation, Milan University, Milan, Italy

j Neuro ICU Fondazione IRCCS Cà Granda Ospedale Maggiore Policlinico Milano, Milan, Italy

${ }^{\mathrm{k}}$ Department of Medical Ethics and Philosophy of Medicine, Erasmus MC - University Medical Centre Rotterdam, Rotterdam, the Netherlands

\section{A R T I C L E I N F O}

Available online $\mathrm{xxxx}$

\section{Keywords:}

Traumatic brain injury

Informed consent

European Union

Ethics

\begin{abstract}
A B S T R A C T
Purpose: Enrolling traumatic brain injury (TBI) patients with an inability to provide informed consent in research is challenging. Alternatives to patient consent are not sufficiently embedded in European and national legislation, which allows procedural variation and bias. We aimed to quantify variations in informed consent policy and practice.

Methods: Variation was explored in the CENTER-TBI study. Policies were reported by using a questionnaire and national legislation. Data on used informed consent procedures were available for 4498 patients from 57 centres across 17 European countries.

Results: Variation in the use of informed consent procedures was found between and within EU member states. Proxy informed consent ( $\mathrm{N}=1377 ; 64 \%)$ was the most frequently used type of consent in the ICU, followed by patient informed consent $(\mathrm{N}=426 ; 20 \%)$ and deferred consent $(\mathrm{N}=334 ; 16 \%)$. Deferred consent was only actively used in 15 centres (26\%), although it was considered valid in 47 centres (82\%).

Conclusions: Alternatives to patient consent are essential for TBI research. While there seems to be concordance amongst national legislations, there is regional variability in institutional practices with respect to the use of different informed consent procedures. Variation could be caused by several reasons, including inconsistencies in clear legislation or knowledge of such legislation amongst researchers.
\end{abstract}

(C) 2020 Published by Elsevier Inc.

\section{Background}

Patient informed consent is one of the basic principles underpinning clinical research. Patients have the right to be informed about a proposed study and should have the opportunity to make an autonomous decision on study participation. It is however impossible to obtain patient informed consent from patients with an acute inability to provide informed consent due to an acute illness such as traumatic brain injury (TBI) [1]. Research with TBI patients is however essential to optimize treatments and improve patient outcome. Therefore, several pragmatic alternatives are available in case patient informed consent could not be obtained [2].

Proxy informed consent is the most frequently used alternative. Close family members or unrelated appointed legally authorised representatives are selected in accordance with applicable national or local regulations. These so-called proxies have the legal right to provide informed consent on behalf of the patient [3]. Proxies are however often unavailable in the acute setting or are unable to make a valid judgment for several other reasons [4-9]. This is especially complicated in emergency research where time is scarce.

To overcome this, some research settings allow an independent physician to decide on behalf of the patient. In many European countries, it is also accepted to include and randomize patients in emergency research settings without prior patient- or proxy informed consent and ask consent for study continuation later (deferred consent procedure) $[3,10]$. Researchers can also use the so-called 'exception from consent' and 'waiver of consent' procedures, which allow study start without prior patient- or proxy informed consent without the requirement of informed consent for study continuation [11,12].

The relative pros and cons of different informed consent procedures have led to substantial regulatory variation within and between European Union (EU) Member States and globally [13,14]. The EU has replaced the Data Protection Directive and the Clinical Trials Directive by the General Data Protection Regulation and the Clinical Trials Regulation to harmonize informed consent procedures [3,15-17]. Unfortunately, neither regulation addresses the specific situations of patients with an acute inability to provide informed consent in detail, and neither clearly differentiates between acute or chronic mental conditions. Although the General Data Protection Regulation provides for exemptions from patient informed consent procedures for observational research by leaving room for national legislation, informed consent in clinical emergency research is not mentioned in national law in $12 \mathrm{EU}$ Member States [13,18].

The lack of clear directions in European and national legislation may be expected to result in substantial practice variation in consent procedures for patients with an acute inability to provide informed consent [19]. The use of different informed consent procedures in international multi-center studies could cause recruitment inefficiency, nonhomogenous patient inclusion, selection bias, asymmetrical randomisation, and limited external validity of study results [20,21]. Clearly, optimization of informed consent procedures and harmonization of regulations is important for future research initiatives. 
The aim of this study is to inform researchers and policymakers on the use and challenges of informed consent procedures in a large prospective observational study including patients with an acute inability to provide informed consent due to TBI. Therefore, we investigated local policy and observed practice of informed consent procedures in the Collaborative-European-Neuro-Trauma-Effectiveness-Research in Traumatic Brain Injury (CENTER-TBI) study [22].

\section{Materials and methods}

\subsection{CENTER-TBI and study sample}

The CENTER-TBI project includes a large prospective observational study on TBI conducted in 63 neurotrauma centres across Europe and Israel. [20-21] CENTER-TBI had a follow up period of 12 to 24 months and required extra blood samples and, in a subpopulation, MRI scans in addition to standard care. For this particular study, we excluded four centres with low inclusion rates (<five patients) and 2 centres from Israel, because we focussed on European centres. All remaining centres $(\mathrm{N}=57)$ from 17 European countries obtained IRB approval and were analyzed.(See Suppl Table 1).

\subsection{Policy: provider profiling and national legislation}

Investigators of each study center completed "Provider Profiling" questionnaires prior to recruitment to the CENTER-TBI Core study. The questionnaires aimed to characterize general healthcare processes and, specifically for this present study, the use of informed consent procedures. (see Suppl file 1). These questions were about the acceptance and use of informed consent procedures in general and not specifically for the CENTER-TBI study. The question mentioning the 'deferred consent/waiver of consent' alternatives was used to assess the possibility of study start without prior informed consent in emergency research and was named deferred consent in this article. Answers explicitly represent a general consensus at the centres, rather than an individuals' preference, in an attempt to capture the actual policy of all study centres. Responses were collected and stored by using a secure online database (QuesGen Systems Incorporated, Burlingame, CA, USA) [23]. Detailed information on the provider profiling questionnaires has been published previously [24]. An additional analysis of national regulations that were applicable at the time of study was performed and compared with the results of the questionnaire and actual observed informed consent procedures [13].

\subsection{Practice: CENTER-TBI core study}

The CENTER-TBI Core study (clinicaltrials.gov NCT02210221; RRID: SCR_015582) was conducted between December 2014 and December 2017 [25]. Enrolment criteria were a clinical diagnosis of TBI, indication for CT-scanning, and presentation to study centre within $24 \mathrm{~h}$ of injury. Approval from an IRB or any other appropriate ethics review body was obtained by all centres and informed consent procedures followed local and national requirements. On enrolment, patients were differentiated by care pathway: ER stratum (discharged from emergency room), Admission stratum (hospital ward), and ICU stratum (admission to the intensive care unit (ICU)). For this study, informed consent practice was pragmatically observed in the ICU stratum ( $\mathrm{N}=2137)$ of CENTER-TBI, since we focussed on patients with an acute inability to provide informed consent. The presence of the inability to provide informed consent was very unlikely in patients from the ER and Admission stratum because nearly all sustained mild TBI and provided informed consent themselves.

Clinical data included details on the type and time of informed consent and were collected and de-identified using a web-based electronic case report form (QuesGen) and stored on a secure database, hosted by the International Neuroinformatics Coordinating Facility (INCF; www. incf.org) in Stockholm, Sweden [26].

\subsection{Analyses}

Data (Version $1 \cdot 0$, released: 01/11/2018) was extracted via the custom-made data access tool Neurobot (http://neurobot.incf.org), developed by INCF. Descriptive statistics were used to obtain frequencies and percentages. For analysis of potential differences between regions we grouped countries into six regions based on the United Nations geo-scheme (See Suppl Table 1). [27] Due to the agreed anonymity of participating sites, it was not always possible to display all differences between countries, as some countries have only 1 or 2 participating sites. Potential differences between centres in one country were analyzed in countries with three or more participating centres. Analyses were performed using R version 3.6.0.

\section{Results}

All 57 participating centres completed the provider profiling questionnaire. The majority was completed by principal investigators and medical professionals $(N=20)$, IRB members $(N=15)$, and staff members $(\mathrm{N}=13)$. (See Suppl Table 2) Most centers were academic hospitals $(91 \%)$ with a designation as Level I trauma centre (68\%). Thirty (53\%) centres had a department of medical ethics and 28 (49\%) had extensive neurotrauma research experience, with five or more research applications over the previous five years. (See Suppl Table 3).

\section{Policy}

Alternatives for patient informed consent were widely accepted. (Table $1 \&$ Fig. 1). Most IRBs allowed the use of proxy informed consent (79\%) for acutely mentally incapacitated patients, while consent by an independent physician was less frequently allowed (37\%). The majority of centers considered deferred consent ( $82 \%$ ) for emergency research to be a valid alternative.

Substantial variation in informed consent policies was noted between regions in Europe. All centres in Northern and Eastern Europe reported prior proxy informed consent to be valid (100\%), in contrast to centres in The Baltic States (75\%), Southern Europe (45\%), the United Kingdom (UK) (89\%) and Western Europe (81\%). Regarding Southern Europe, especially Italian centers (62\%) reported proxy informed consent to be invalid. (See Suppl Table 4).

Acceptance of consent by an independent physician was lower (37\%) and variable across European regions. (See fig. 1 \& Suppl Table 4) It was especially considered valid in Germany (100\%), the UK (89\%), and Spain (67\%). None of the centers from The Netherlands, Italy and Norway reported this alternative to be valid, while other countries were inconsistent. (see Suppl Table 5).

The use of the deferred consent procedure was reported valid by most centers in most regions, except Eastern Europe. (see Suppl Table 4) When reported valid, it was mostly regulated by IRB approval $(\mathrm{N}=36)$ or by law $(\mathrm{N}=11)$. Of countries with $\geq 3$ centres, all mentioned that the procedure was valid. (see Suppl Table 5).

Table 1

Number of study centres (\%) that allow the use of an informed consent procedure in acutely mentally incapacitated patients.

\begin{tabular}{llll}
\hline Informed consent procedure & Yes & No & Unknown \\
& N (\%) & N (\%) & N (\%) \\
\hline Proxy informed consent & $45(79)$ & $11(19)$ & $1(2)$ \\
Consent by an independent physician & $21(37)$ & $30(53)$ & $6(10)$ \\
Deferred consent & $47(83)$ & $7(12)$ & $3(5)$ \\
\hline
\end{tabular}




\section{POLICY patient informed consent allowed}

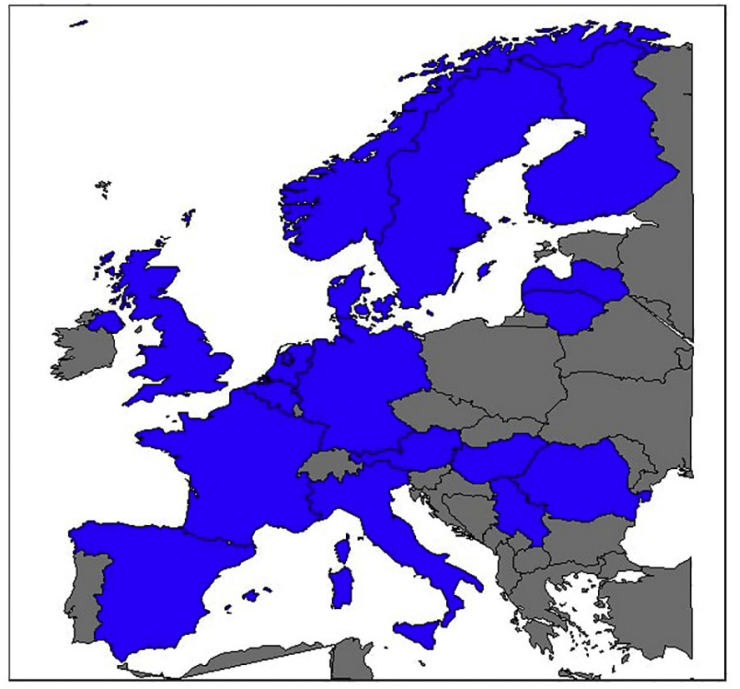

POLICY proxy informed consent allowed

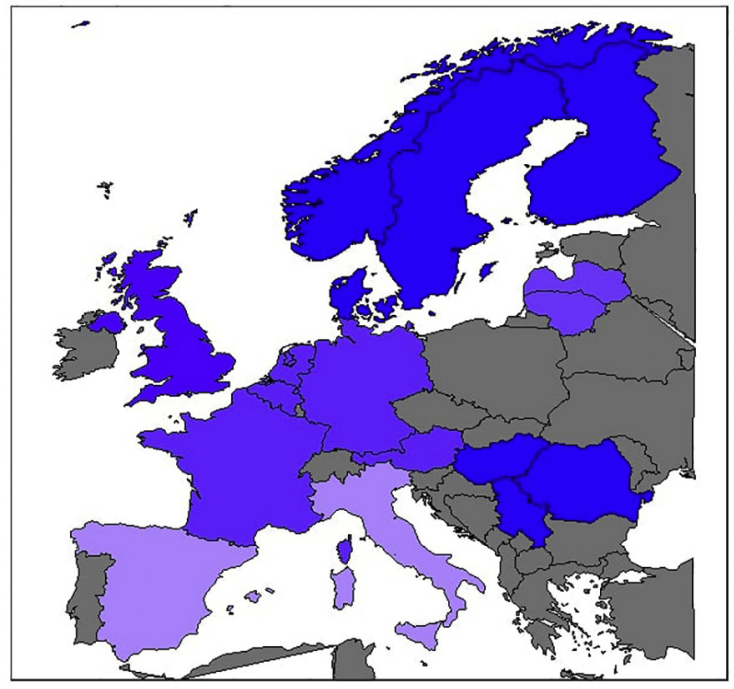

POLICY deferred consent allowed

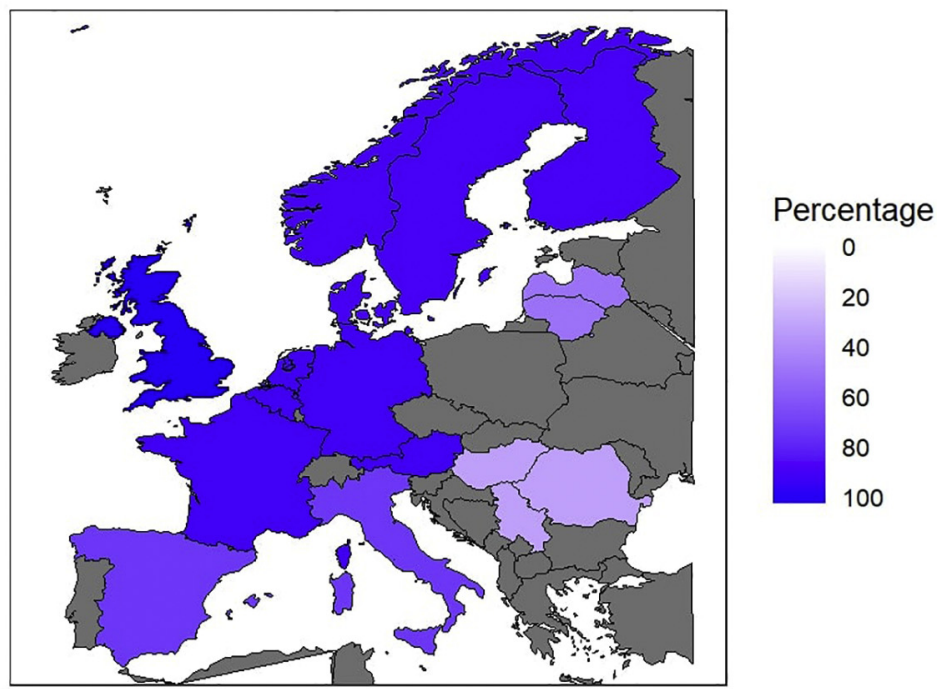

Percentage

0
20
40
60
80
100

Percentage

0
20
40
60
80
100 
Table 2

Number of patients (\%) and type of used informed consent procedure per stratum in the CENTER-TBI study.

\begin{tabular}{|c|c|c|c|}
\hline Consent type / Stratum & $\mathrm{ER}(\mathrm{N}=844,19 \%)$ & Admission $(\mathrm{N}=1517,34 \%)$ & $\mathrm{ICU}(\mathrm{N}=2137,48 \%)$ \\
\hline Patient informed consent $(\mathrm{N}=2497,56 \%)$ & $805(95)$ & $1266(83)$ & $426(20)$ \\
\hline Proxy informed consent $(\mathrm{N}=1635,36 \%)$ & $35(4)$ & $223(15)$ & $1377(64)$ \\
\hline Deferred consent $(\mathrm{N}=366,8 \%)$ & $4(0 \cdot 5)$ & $28(2)$ & $334(16)$ \\
\hline
\end{tabular}

\section{Practice}

\subsection{Overall practice}

All participating centres $(\mathrm{N}=57)$ included 4498 patients. Most patients were admitted to the ICU stratum $(\mathrm{N}=2137 ; 48 \%)$ followed by the Admission stratum $(\mathrm{N}=1517 ; 34 \%)$ and the ER stratum $(\mathrm{N}=$ $844 ; 19 \%)$. Overall, patient informed consent $(N=2497 ; 56 \%)$ was the most frequently used type of consent, followed by proxy informed consent $(\mathrm{N}=1635 ; 36 \%)$ and deferred consent $(\mathrm{N}=366 ; 8 \%)$ The use of patient informed consent was lower for patients requiring ICU admission ( $\mathrm{N}=426 ; 20 \%$ ) compared to patients requiring admission to the ward $(\mathrm{N}=1266 ; 83 \%)$. (Table 2$)$.

\subsection{Practice in ICU stratum}

Proxy informed consent ( $\mathrm{N}=1377 ; 64 \%)$ was the most frequently used type of consent in the ICU, followed by patient informed consent ( $N=426 ; 20 \%)$ and deferred consent $(\mathrm{N}=334 ; 16 \%$ ) (Table 3). Proxy informed consent was most frequently used in the UK (96\%), Southern Europe (80\%) and The Baltic States (76\%), and less frequently in Northern (56\%) and Western Europe (49\%). In contrast, deferred consent was most frequently used in Northern (19\%) and Western Europe (25\%) but infrequently in the UK (0.3\%) and the Baltic States (3\%) (Table 3). Seven countries (41\%) did not use deferred consent. Austria did not use proxy informed consent, but showed the highest number of deferred consents instead (65\%). (see Suppl Table 6).

\section{Comparison of policy and practice}

Proxy informed consent and deferred consent procedures are accepted by national legislation of all displayed countries [13,28,29]. (Table 4) Some centers however reported proxy or deferred consent procedures to be not accepted. In addition, there was variation between accepted procedures and actually used informed consent procedures. Italy for instance reported a low rate of proxy informed consent acceptance and a high enrolment rate using proxy informed consent.

When also including countries ( $\leq 3$ centres) that could not be displayed, the use of deferred consent in emergency situations was allowed in 10 out of 17 countries. The procedure was not mentioned in national legislation in 6 countries. In the questionnaire, 47 (82\%) of the participating centres reported that it was possible to include patients with an acute inability to provide informed consent by using deferred consent. In practice, only 15 centres from seven countries were responsible for $99 \%(\mathrm{~N}=330)$ of the deferred consent cases in the ICU.

\section{Discussion}

Patient informed consent alternatives like proxy informed consent, deferred consent and independent physician consent were widely used in the CENTER-TBI study and were essential to include ICU admitted TBI patients with an acute inability to provide informed consent. Alternatives to patient informed consent are essential in TBI research. Only $20 \%$ of ICU patients provided patient informed consent. This study found substantial between and within-country variation in reported accepted informed consent policies and actually used informed consent procedures. Variation could be caused by several reasons and could indicate that either clear national or European legislation is unavailable or that knowledge of such legislation may be inconsistent amongst clinicians and researchers.

The number of patient informed consent ( $\mathrm{N}=2497 ; 56 \%$ ) observed in the CENTER-TBI core study was higher than expected. This was partly due to the large number of patients in the ER and Admission strata ( $>95 \%$ with mild TBI) that were able to provide informed consent (87\%). In addition, many patients in the ICU stratum had mild TBI (36\%) [27]. This could explain the high number of patient informed consents (20\%) in the ICU, but it is also possible that study personnel wrongly considered a patient to have the ability to provide patient informed consent. The CENTER-TBI study did not use or document any assessment of a patients' ability to provide informed consent. Although assessment methods are available and used in some studies, they have important limitations [30,31]. It is important that researchers formally assess the ability to provide informed consent in all patients when possible. Especially in patients with a possible episode of an acute inability to provide informed consent. This assessment should ideally be recorded in the case report form to guarantee the validity of patient informed consent.

Alternatives for patient informed consent allowed the inclusion of $80 \%$ of ICU stratum patients. Overall, proxy informed consent was the most frequently used alternative. Although it was not always reported to be an accepted informed consent policy for mentally incapacitated patients, it was an accepted procedure by all national laws. Proxies usually prefer to be involved in decision-making, but proxy informed consent has several important limitations [32]. Several studies report substantial discrepancies between patients and proxies and conclude that proxies are poor surrogate decision-makers [7-9,33]. In addition, proxies are not always present in emergency situations, or are too overwhelmed by the stressful situation to provide valid proxy informed consent $[34,35]$. Researchers and clinicians should be aware of the many factors that are important in the process of informed consent [36].

Fortunately, it was also possible to include patients by using deferred consent when it was impossible to obtain prior patient or proxy informed consent. A total of 45 centres (79\%) from ten countries,

Table 3

Number of patients (\%) and type of used informed consent procedures in the ICU stratum per region.

\begin{tabular}{|c|c|c|c|c|c|c|c|}
\hline Answers|Regions & $\begin{array}{l}\text { Sample Total } \\
(\mathrm{N}=2137)\end{array}$ & $\begin{array}{l}\text { Baltic States } \\
(\mathrm{N}=33)\end{array}$ & $\begin{array}{l}\text { Eastern Europe } \\
(\mathrm{N}=33)\end{array}$ & $\begin{array}{l}\text { Northern Europe } \\
(\mathrm{N}=391)\end{array}$ & $\begin{array}{l}\text { Southern Europe } \\
(\mathrm{N}=546)\end{array}$ & $\begin{array}{l}\text { United Kingdom } \\
(\mathrm{N}=271)\end{array}$ & $\begin{array}{l}\text { Western Europe } \\
(\mathrm{N}=863)\end{array}$ \\
\hline Patient informed consent & $426(20)$ & $7(21)$ & $11(33)$ & $97(25)$ & $75(14)$ & $10(4)$ & $226(26)$ \\
\hline Proxy informed consent & $1377(64)$ & $25(76)$ & $20(61)$ & $219(56)$ & $433(79)$ & $260(96)$ & $420(49)$ \\
\hline Deferred consent & $334(16)$ & $1(3)$ & $2(6)$ & $75(19)$ & $38(7)$ & $1(0 \cdot 3)$ & $217(25)$ \\
\hline
\end{tabular}


Table 4

Comparison of observed practice, national legislation and reported policy regarding informed consent procedures in the CENTER-TBI ICU stratum.

\begin{tabular}{|c|c|c|c|c|c|c|c|c|}
\hline Country & $\begin{array}{l}\text { Number } \\
\text { of } \\
\text { centers } \\
\text { per } \\
\text { country }\end{array}$ & $\begin{array}{l}\text { Patients } \\
\text { included } \\
\text { using } \\
\text { patient } \\
\text { informed } \\
\text { consent ( } \mathrm{N} \\
(\%) \text { ) }\end{array}$ & $\begin{array}{l}\text { Proxy informed } \\
\text { consent procedures } \\
\text { accepted according to } \\
\text { national legislation? } \\
\text { [13] }\end{array}$ & $\begin{array}{l}\text { Number of centers (\%) } \\
\text { accepting proxy } \\
\text { informed consent } \\
\text { according to provider } \\
\text { profiling }\end{array}$ & $\begin{array}{l}\text { Patients } \\
\text { included } \\
\text { using proxy } \\
\text { informed } \\
\text { consent } \\
(\mathrm{N}(\%))\end{array}$ & $\begin{array}{l}\text { Deferred consent } \\
\text { accepted in } \\
\text { emergency } \\
\text { research according to } \\
\text { national legislation? } \\
\text { [13] }\end{array}$ & $\begin{array}{l}\text { Number of centers (\%) } \\
\text { accepting deferred } \\
\text { consent } \\
\text { in emergency research } \\
\text { according to provider } \\
\text { profiling }\end{array}$ & $\begin{array}{l}\text { Patients } \\
\text { included } \\
\text { using } \\
\text { deferred } \\
\text { consent } \\
(\mathrm{N}(\%))\end{array}$ \\
\hline Belgium & 4 & $71(37)$ & Yes & $4(100)$ & $122(63)$ & Yes & $4(100)$ & $0(0)$ \\
\hline France & 5 & $25(22)$ & Yes & $5(100)$ & $90(78)$ & Yes & $5(100)$ & $0(0)$ \\
\hline Germany & 4 & $24(28)$ & Yes & $2(50)$ & $54(62)$ & Yes & $3(75)$ & $9(10)$ \\
\hline Italy & 8 & $34(10)$ & Yes & $3(37)$ & $279(79)$ & Yes & $5(63)$ & $38(11)$ \\
\hline Netherlands & 7 & 68 (19) & Yes & $6(86)$ & $154(43)$ & Yes & $6(86)$ & $137(38)$ \\
\hline Norway & 3 & $33(20)$ & Yes [28] & $3(100)$ & $94(58)$ & Yes [29] & $3(100)$ & $36(22)$ \\
\hline Spain & 3 & $41(21)$ & Yes & $2(67)$ & $154(79)$ & Not mentioned & $3(100)$ & $0(0)$ \\
\hline UK & 9 & $10(4)$ & Yes & $8(89)$ & $260(96)$ & Yes & $9(100)$ & $1(0.4)$ \\
\hline Total & 43 & 306 & & 33 & 1207 & & 38 & 221 \\
\hline
\end{tabular}

according to national law, or 47 centres (82\%), according to reported policies, were allowed to use this procedure. Nonetheless, only 15 centres (26\%) actively ( $>2$ inclusions) used it. There are multiple explanations for this discrepancy. First, the use of deferred consent might be accepted in national legislation, but local IRBs may not have authorised it for the CENTER-TBI study. Also, the use of deferred consent is not ethically neutral and the acceptance by IRBs, healthcare providers, patients and relatives differ substantially [37-42]. Second, deferred consent was authorised as a valid, but its use was not required because proxy or independent physician consent were used. Last, it is also possible that local researchers were unaware of the possibility of deferred consent.

Current European regulations include The Data Protection Directive and the Clinical Trials Directive, which were applicable at the time when patients were included in CENTER-TBI, are or will be superseded by the General Data Protection Regulation and the Clinical Trial Regulation respectively. However, since the General Data Protection Regulation does not apply to anonymized data and alternatives to patient informed consent are left to the legislation of Member States, large improvements in harmonization are not expected. [19,43] The Clinical Trials Regulation does state that patient informed consent may be deferred in some specific situation and might thereby cause an increase in the use of deferred consent. [17,19,44-46]

There is a lack of clear regulations on emergency research in mentally incapacitated patients and lack of harmonization regarding informed consent procedures in European Neurotrauma centres. Performing multinational trials is challenging when variations in acceptance of alternatives for patient informed consent exist [14,47]. Potential issues not only include IRB processing and patient recruitment inefficiency and therefore study delay, but also non-homogenous patient inclusion, selection bias, asymmetrical randomisation, and limited external validity of study results. [20,21] Although informed consent procedures are bound by national laws, institutional regulations and cultural factors, it could be beneficial for future research initiatives to harmonize procedures and regulations.

This study has several limitations. First, the majority of the participating centres were academic centres specialized in research and neurotrauma resulting in a possible selection bias. Second, by pragmatically focusing on patients from the ICU stratum with the highest likelihood of an inability to provide informed consent, we might have missed a few patients that were included in the ER or ward stratum. Unfortunately, there was no registered formal assessment of the ability to provide informed consent that could have been used to identify patients. Third, in addition to an analysis of national laws, reported informed consent policies were based on the provider profiling questionnaire rather than on actual policies. Although most responses were provided by seniors, the discrepancies could be caused by provider profiling errors due to variable individual understanding of actual policies and/or regulations. It could however also reflect the centres' general consensus or
IRB specific directives rather than national juridical policies. Fourth, it is important to bear in mind that CENTER-TBI is an observational study, although IRBs in three countries considered it to be an interventional study as blood samples were requested. Results on consent policy and practice might be different for interventional studies or randomized controlled trial. This is because the consequences of participation might be bigger and effective retrospective refusal of study participation is not possible as study interventions have already taken place. Although our data are derived from a patient population with TBI, the identified problems and insights have relevance for other conditions that could cause an inability to provide informed consent.

\section{Conclusion}

Alternatives to patient informed consent are essential for studies including TBI patients with an acute inability to provide informed consent. The substantial variation in reported and used informed consent procedures in Europe could be caused by several reasons and could indicate that clear national or European legislation is unavailable or that knowledge of such legislation may be inconsistent amongst clinicians and researchers. Future research initiatives could benefit from clear and harmonized regulations for this subcategory of patients.

\section{Ethics approval and consent to participate}

CENTER-TBI has received ethical approval by IRBs of all participating centres and informed consent for participation has been collected accordingly. Participating centres have given consent by completing the questionnaire.

\section{Consent for publication}

Not applicable.

\section{Availability of data and materials}

There are legal constraints that prohibit us from making the data publicly available. Since there are only a limited number of centres per country included in this study (for two countries only one centre), data will be identifiable. Readers may contact Dr. Erwin J. O. Kompanje (e.j.o.kompanje@erasmusmc.nl) for reasonable requests for the data.

\section{Funding}

CENTER-TBI was supported by the European Union 7th Framework program (EC grant 602150). Additional funding was obtained from the Hannelore Kohl Stiftung (Germany), from OneMind (USA) and from Integra LifeSciences Corporation (USA). David K. Menon was 
supported by a Senior Investigator Award from the National Institute for Health Research (UK).

\section{Authors' contributions}

RW and JD contributed equally to the study. RW, JD and MT analyzed the data and drafted the manuscript, and the supplementary tables. All coauthors gave feedback on the manuscript. EJOK supervised the project. All coauthors were involved in the design of the survey and the distribution of the survey. All coauthors gave feedback on (and approved) the final version of the manuscript.

Supplementary data to this article can be found online at https://doi. org/10.1016/j.jcrc.2020.05.004.

\section{Declaration of Competing Interest}

The authors declare that they have no competing interests.

\section{References}

[1] Association WM. Declaration of Helsinki - Ethical Principles for Medical Research Involving Human Subjects. 2018; 2019.

[2] Grady C, Cummings SR, Rowbotham MC, McConnell MV, Ashley EA, Kang G. Informed consent. N Engl J Med 2017;376:856-67. https://doi.org/10.1056/ NEJMra1603773.

[3] Regulation (EU). No 536/2014 of the European Parliament and of the Council of 16 April 2014 on clinical trials on medicinal products for human use, and repealing Directive 2001/20/EC OJ L158:1-76; 2014.

[4] Mirr MP. Decisions made by family members of patients with severe head injury. ACCN 1991;2:242-51. https://doi.org/10.4037/15597768-1991-2009.

[5] Gigon F, Merlani P, Chenaud C, Ricou B. ICU research: the impact of invasiveness on informed consent. Intens Care Med 2013;39(7):1282-9. https://doi.org/10.1007/ s00134-013-2908-X.

[6] Abramson NS, Meisel A, Safar P. Deferred consent: a new approach for resuscitation research on comatose patients. JAMA 1986;255:2466-71. https://doi.org/10.1001/ jama.255.18.2466.

[7] Wrigley A. Proxy consent: moral authority misconceived. J Med Ethics 2007;33: 527-31. https://doi.org/10.1136/jme.2006.019711.

[8] Ciroldi M, Cariou A, Adrie C, Annane D, Castelain V, Cohen Y, et al. Ability of family members to predict patient's consent to critical care research. Intensive Care Med 2007;33:807-13. https://doi.org/10.1007/s00134-007-0582-6.

[9] Shalowitz DI, Garrett-Mayer E, Wendler D. The accuracy of surrogate decision makers: a systematic review. Arch Intern Med 2006;166:493-7. https://doi.org/10. 1001/archinte.166.5.493.

[10] Jansen TC, Kompanje EJO, Bakker J. Deferred proxy consent in emergency critical care research: Ethically valid and practically feasible. Crit Care Med 2009;37 (Suppl):65-8. https://doi.org/10.1097/CCM.0b013e3181920851.

[11] Department of Health and Human Services F and DA. Protection of human subjects; informed consent and waiver of informed consent requirements in certain emergency Research; final rules. Fed Regist 1996;61(192):51498-531.

[12] U.S. Food and Drug Administration. Sec. 50.24. Exception From Informed Consent Requirements for Emergency Research. Code Fed Regul Title 21, Vol I, Subchapter A, Part 50, Subpart B; 2019.

[13] Kompanje EJO, Maas AIR, Menon DK, Kesecioglu J. Medical research in emergency research in the European Union member states: tensions between theory and practice. Intens Care Med 2014;40:496-503. https://doi.org/10.1007/s00134-0143243-6.

[14] Van Belle G, Mentzelopoulos SD, Aufderheide T, May S, Nichol G. International variation in policies and practices related to informed consent in acute cardiovascular research: results from a 44 country survey. Resuscitation 2015;91:76-83. https:// doi.org/10.1016/j.resuscitation.2014.11.029.

[15] Directive 2001/20/EC of the European Parliament and of the Council of 4 April 2001. On the Approximation of the Laws, Regulations and Administrative Provisions of the Member States Relating to the Implementation of Good Clinical Practice in the Conduct of Clinical Trials on Medicinal Products for Human Use. OJ L121:34; 2001.

[16] Directive 95/46/EC of the European Parliament and of the Council of 24 October 1995. On the Protection of Individuals With Regard to the Processing of Personal Data and on the Free Movement of Such Data OJ L281:31-50; 1995.

[17] Regulation (EU) No 2016/679 of the European Parliament and of the Council of 27 April 2016. On the Protection of Natural Persons With Regard to the Processing of Personal Data and on the Free Movement of Such Data, and Repealing Directive 95/46/EC (General Data Protection Regulation) OJ L119:1-88; 2016.

[18] European Union GDPR. Processing of Special Categories of Personal Data. O] L119:Ch. 2 Art. 9; 2016.
[19] Timmers M, Van Veen EB, Maas AIR, Kompanje EJO. Will the EU data protection regulation 2016/679 inhibit critical care research? Med Law Rev 2019;27:59-78. https://doi.org/10.1093/medlaw/fwy023.

[20] Ecarnot F, Quenot JP, Besch G, Piton G. Ethical challenges involved in obtaining consent for research from patients hospitalized in the intensive care unit. Ann Transl Med 2017;5(suppl):S41. https://doi.org/10.21037/atm.2017.04.42.

[21] Burns KEA, Zubrinich C, Tan W, Raptis S, Xiong W, Smith O, et al. Research recruitment practices and critically ill patients. A multicenter, cross-sectional study (the consent study). Am J Respir Crit Care Med 2013;187:1212-7. https://doi.org/10 1164/rccm.201208-15370C.

[22] Maas AIR, Menon DK, Steyerberg EW, Citerio G, Lecky F, Manley GT, et al. Collaborative European NeuroTrauma effectiveness Research in traumatic brain injury (CENTER-TBI): a prospective longitudinal observational study. Neurosurgery 2015;76(1): 67-80. https://doi.org/10.1227/NEU.0000000000000575.

[23] QuesGen Systems, Inc. Available from https://www.quesgen.com/; 2019, Accessed date: 8 October 2019.

[24] Cnossen MC, Polinder S, Lingsma HF, Maas AI, Menon D, Steyerberg EW. Variation in structure and process of care in traumatic brain injury: provider profiles of European neurotrauma centers participating in the CENTER-TBI study. Plos One 2016;11:1. https://doi.org/10.1371/journal.pone.0161367.

[25] Steyerberg E, Wiegers E, Sewalt C, Buki A, Citerio G, De Keyser V, et al. Case-mix, care pathways, and outcomes in patients with traumatic brain injury in CENTER-TBI: a European prospective, multicentre, longitudinal, cohort study. Lancet Neurol 2019; 18(10):923-34. https://doi.org/10.1016/S1474-4422(19)30232-7.

[26] International Neuroinformatics Coordinating Facility (INCF). Available at https:// www.incf.org/; 2019, Accessed date: 8 October 2019.

[27] United Nations. Standard Country or Area Codes for Statistical Use (M49). Available from: https://unstats.un.org/unsd/methodology/m49/; 2019, Accessed date: 8 October 2019.

[28] Convention on the Protection of Human Rights and Human Dignity in Connection With the Application of Biology and Medicine: Convention on Human Rights and Biomedicine - ETS no. 164. Chapter 5, Article 17.6; 1997.

[29] Convention on the Protection of Human Rights and Human Dignity in Connection With the Application of Biology and Medicine: Convention on Human Rights and Biomedicine - ETS no. 164. Chapter 5, Article 17.2; 1997.

[30] Johnson-Greene D. Informed consent issues in traumatic brain injury research: current status of capacity assessment and recommendations for safeguards. J Head Trauma Rehabil 2010;25:145-50. https://doi.org/10.1097/HTR.0b013e3181d8287d.

[31] Feng KL, Person C, Phillips-Sabol J, Williams B, Cai C, Jacobs AN, et al. Comparison between a standardized questionnaire and expert clinicians for capacity assessment in stroke clinical trials. Stroke 2014;45:e229-32. https://doi.org/10.1161/STROKEAHA. 114.006395.

[32] Barrett KA, Ferguson ND, Athaide V, Cook DJ, Friedrich JO, McDonald E, et al. Surrogate decision makers' attitudes towards research decision making for critically ill patients. Intensive Care Med 2012;38:1616-23. https://doi.org/10.1007/s00134-0122625-x.

[33] Newman JT, Smart A, Reese TR, Williams A, Moss M. Surrogate and patient discrepancy regarding consent for critical care research. Crit Care Med 2012;40:2584-90.

[34] Shepherd V, Hood K, Sheehan M, Griffith R, Wood F 'It's a tough decision': a qualitative study of proxy decision-making for research involving adults who lack capacity to consent in UK. Age Ageing 2019;48:903-9. https://doi.org/10.1093/ageing/ afz115.

[35] Long B, Clark L, Cook P. Surrogate decision making for patients with severe traumatic brain injury. J Trauma Nurs 2011;18:204-12. https://doi.org/10.1097/JTN Ob013e31823a453a.

[36] Hall DE, Prochazka AV, Fink AS. Informed consent for clinical treatment. CMAJ 2012; 184(5):533-40. https://doi.org/10.1503/cmaj.112120.

[37] Topolovec-Vranic J, Santos M, Baker AJ, Smith OM, Burns KE. Deferred consent in minimal-risk study involving critically ill subarachnoid hemorrhage patients. Can Respr J 2014;21:293-6. https://doi.org/10.1155/2014/719270.

[38] Honarmand K, Belley-Cote EP, Ulic D, Khalifa A, Gibson A, McClure G, et al. The deferred consent model in prospective observational study evaluating myocardial injury in the intensive care unit. J Intensive Care Med 2018;33:475-80. https://doi org/10.1177/0885066616680772.

[39] Shamy MCF, Dewar B, Chevrier S, Wang CQ, Page S, Goyal M, et al. Deferral of consent in acute stroke trials. Lessons from the ESCAPE trial. Stroke 2019;50:1017-20. https://doi.org/10.1161/STROKEAHA.118.024096.

[40] Woolfall K, Frith L, Gamble C, Gilbert R, Mok Q, Young B, et al. How parents and practitioners experience research without prior consent (deferred consent) for emergency research involving children with life threatening conditions: a mixed method survey. BMJ Open 2015;5:e008522. https://doi.org/10.1136/bmjopen2015-008522.

[41] Eltorki M, Uleryk E, Freedman SB. Waiver of informed consent in pediatric resuscitation research: a systematic review. Ac Emerg Med 2013;20:822-34. https://doi.org/ 10.1111/acem.12180.

[42] Duffett M, Burns KE, Kho ME, Lauzier F, Meade MO, Arnold DM, et al. Consent in critical care trials: a survey of Canadian research ethics boards and critical care researchers. J Crit Care 2011;26. https://doi.org/10.1016/j.jcrc.2010.12.009 (533. e11-533.e22).

[43] Chico V. The impact of the general data protection regulation on health research. $\mathrm{Br}$ Med Bull 2018;128:109-18. https://doi.org/10.1093/bmb/ldy038. 
[44] Rumbold JM, Pierscionek B. The effect of the general data protection regulation on medical research. J Med Internet Res 2017;19(2):e47. https://doi.org/10.2196/jmir. 7108.

[45] Clarke N, Vale G, Reeves EP, Kirwan M, Smith D, Farell M. GDPR: an impediment to research? Ir J Med Sci 2019;188(4):1129-35. https://doi.org/10.1007/s11845-01901980-2.
[46] Van Veen EB. Observational health research in Europe: understanding the general data protection regulation and underlying debate. Eur J Cancer 2018;104:70-80. https://doi.org/10.1016/j.ejca.2018.09.032.

[47] Tisherman SA, Powell JL, Schmidt TA, Aufderheide TP, Kudenchuk PJ, Spence J, et al. Regulatory challenges for the resuscitation outcomes consortium. Circulation 2008; 118:1585-92. https://doi.org/10.1161/CIRCULATIONAHA.107.764084. 